\title{
Sobre a baritina de Araxá
}

\author{
(5 figunas no texto)
}

\author{
R. R. Franco
}

As jazidas de Baritina existentes no Brasil se distribuem por differentes pontos do Paiz, sendo que algumas estão hoje em exploração e outras apenas localisadas. As principaes que podemos mencionar estão situadas nos Estados de Minas Geraes, Bahia, São Paulo e Amazonas. Em Minas Geraes: nas localidades de Antonio Pereira (proximidades de Ouro Preto), Ojó (Ouro Preto), Uberaba e estancia do Barreiro (Araxá). Na Bahia: nos municipios de Jussiape, Senhor Bom Jesus dos Meiras e Minas do Rio de Contas. Em São Paulo: na Serra do Serrote (estrada de Rodagem Juquiá - Registro).

ANDRADE JUNior (1) e DJalma Guimarães (2) que estudaram minuciosamente a região do Araxá sob o ponto de vista geologico, chegaram à conclusão de que esta região é constituida de sedimentos metarnorficos da Série de Minas. O mesmo concluiu Octavio Barbosa. (3) Outros autores como Avelino Ignacio de Oliveira (4) e OTHON HENRY LEONARDos (5) que fizeram estudos de natureza diversas são de identico parecer. A rocha que aflora no Bar-

(1) Andrade Junior - " Aguas Mineraes Boletim n. 9 do S. G. M. B. $(1925)$

(2i) DJalma Guimarães - Aguas Mineraes" Boletim n. 9 do S. G. M. B. $(1925)$.

(3) Octayio Barbosa - Geolcgia do Municipio de Araxá (M. Geraes).

(4) Avelino Ignacio de Oliveira - Baritina em Araxá (M. Geraes). Avulso n. 10 (1936) do Departamento N. P M. Ministerio da Agricultura.

(5) Othon Henry Leonardos - (Bario - Avulso n. 2 (1934) do S. Fomento e Producão Mineral. 
reiro e que se acha infiltrada pela baritina é um quartzito da Série de Minas, o que confirmamos pelas observações proprias feitas "in loco" e pelo exame miscroscópico.

Dada a falta de trabalhos morfologicos e cristalograficos sobre a baritina do Brasil, propuzemo-nos a inicial-os com o relativo áquele do Araxá.

Por gentileza do Sr. Demetrio Zema, chefe do serviço de extração nos foi possivel pesquisar uma das jazidas porquanto a outra (Fazenda dos Agudos) já estava abandonada e soterradas em parte as escavações. A baritina encontrada acha-se sob a forma de veios com aspectos diferentes: espáticos, microgranulares e compactos que se ramificam em tôdas as direções, sendo de espessuras variadas. Em lugares onde os veios se alargam encontramse grandes geodos e ainda nódulos de extrutura radiada que preenchem quasi totalmente as cavidades.

$$
* * *
$$

Os cristais, geralmente bem formados, atapetam os veios e cavidades, razão pela qual, raramente se encontram cristais biterminados. Associados a eles:

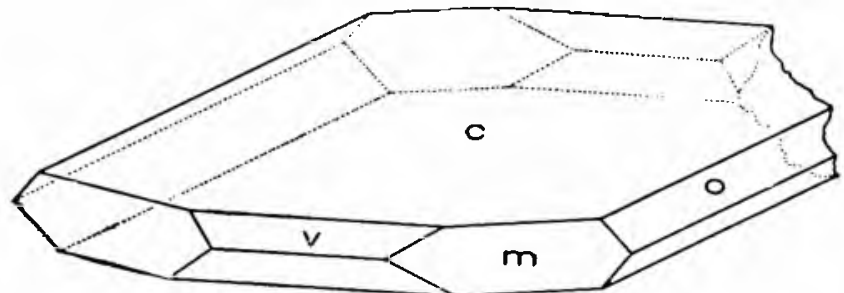

Fig. 1 notam-se grânulos de limonita que dão uma coloração avermelhada ás paredes dos veios. Os cristais são de tamanhos variados alcançando os mais desenvolvidos $\mathbf{5}$ a $6 \mathrm{cms}$. na sua maior dimensão. Dotados geralmente de uma côr levemente esverdeada encontram-se tambem blocos de baritina de forte coloração amarela, devido a traços de limonita. São brilhantes e a maioria de brilho vitreo.

Os habitus dos cristais não são muito variados, o que se pode explicar, admitindo condições de equilibrio fisico-quimico constantes no ambiente de cristalisação. O que predomina é o tabular com desenvolvimento preponderante do pinacoide $\{001\}$, conforme figuras

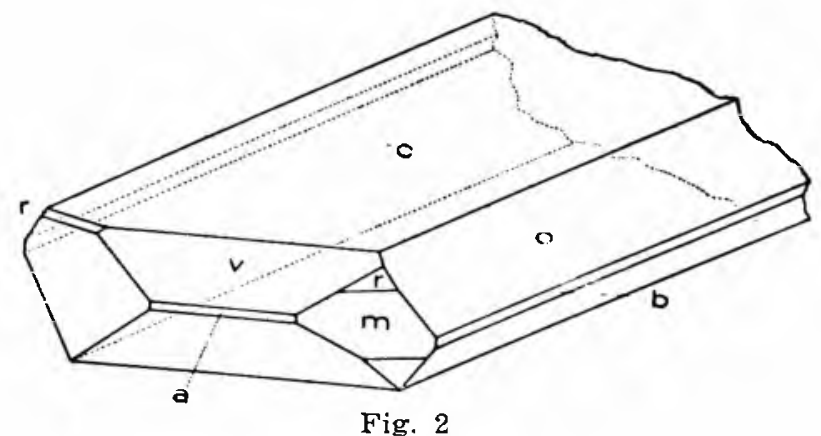
(1), (2), (3). Segue-se o habitus prismático com maior desenvolvimento dos prismas $\{011\}$ e $\{102\}$, fig. (4). Em certos 
casos, os prismáticos são alongados na direção do eixo $x$ e $€ \mathrm{~m}$ outros na direção do eixo do $y$. Em alguns, ha um desenvolvimento aproximadamente igual das (001) e (011) e o cristal

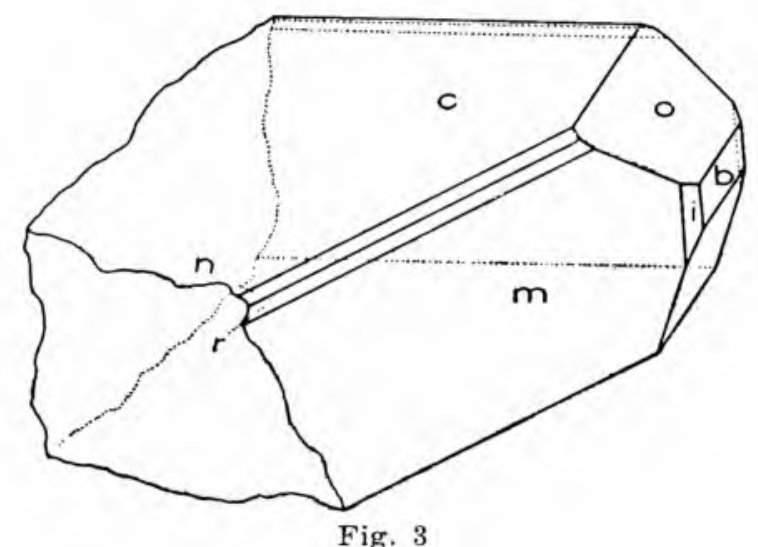

fica com a forma pseudohexagonal, fig. (5) aproximando-se muito do habitus da celestina.

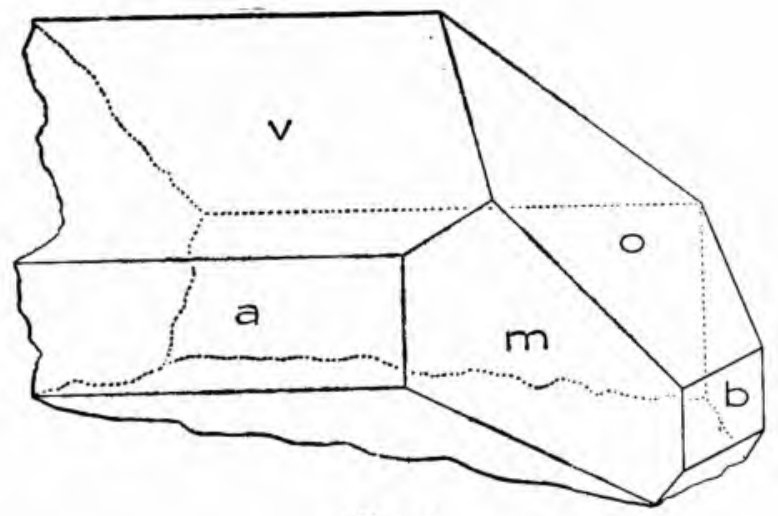

Fyg. 4

As formas encontradas são as seguintes:

$$
\begin{aligned}
& \mathrm{a}\{100\}, \operatorname{m}\{110\}, \mathrm{i}\{130\} \\
& \mathrm{b}\{010\}, \mathrm{o}\{011\} \\
& \mathrm{c}\{001\}, \mathrm{v}\{102\} \\
& \mathrm{r}\{111\}, \mathrm{s}\{223\}, \mathrm{n}\{113\}
\end{aligned}
$$

conforme a projeção anexa, fig. 6 .

A (001) é constante e algumas vêzes bem estriada. Estas estrias são paralelas ás faces (110) e correspondem aos traços de 
clivagem, o que verificamos facilmente. As (011), (102) e (110) são muito brilhantes e de aspecto mais ou menos uniforme. A (130) foi encontrada algumas vêzes, mas pouco desenvolvida e quasi imperceptivel. Seguem-se as faces de piramides (111), (223) e (113) que se mostram sempre muito finas mas oferecendo belo reflexo.

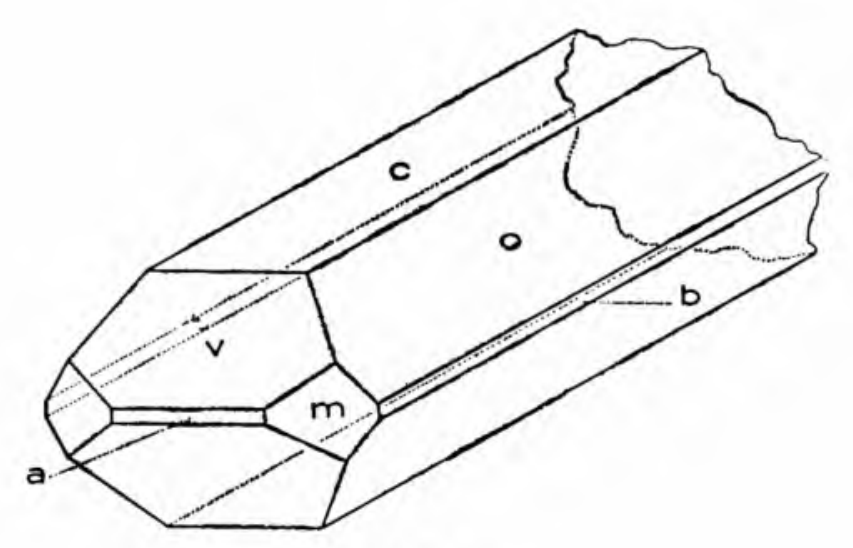

Fig. 5

Anotamos abaixo as combinações encontradas:

(Fig. 1) $\mathrm{m}\{110\}, \mathrm{o}\{011\} \mathrm{c}\{001\}, \mathrm{v}\{102\}$,

(Fig, 2) $\operatorname{m}\{110\}, o\{011\}, c\{001\}, v\{102\}, a\{100\}$, b $\{010\}, r\{111\}$

(Fig. 3) $\mathrm{m}\{110\}, \mathrm{o}\{011\}, \mathrm{c}\{001\}, \mathrm{n}\{113\}, \mathrm{r}\{111\}, \mathrm{i}\{130\}$

(Fig. 4) $\mathrm{m}\{110\}, \mathrm{o}\{011\}, \mathrm{v}\{102\}$ a $\{100\}, \mathrm{b}\{010\}$,

(Fig. 5) $\mathrm{m}\{110\}, \mathrm{o}\{011\}, \mathrm{c}\{001\}, \mathrm{v}\{102\}$ a $\{100\}, \mathrm{b}\{010\}$

As faces $(100),(110),(001)$ e (011) permitiram boas medidas e servindo-nos dos angulos (100) : (110) e (001) : (011) calculamos a seguinte relação parametrica:

$$
\mathrm{a}: \mathrm{b}: \mathrm{c}=0,8156: 1: 1,3123
$$

Esta relação se aproxima muito dos valores encontrados por KoLB (6) para a baritina de Dufton na Inglaterra,

(6) KoLB - (B. de Dufton - Inglaterra) Z. Kryst. 49, 14 - 61, (1911). 


$$
\mathrm{a}: \mathrm{b}: \mathrm{c}=0,8155: 1: 1,3125
$$

e daqueles encontrados por E. ARTINI (7) para a baritina de Aquadurotal na Italia,

$$
\mathrm{a}: \mathrm{b}: \mathrm{c}=0,8156: 1: 1,3121
$$

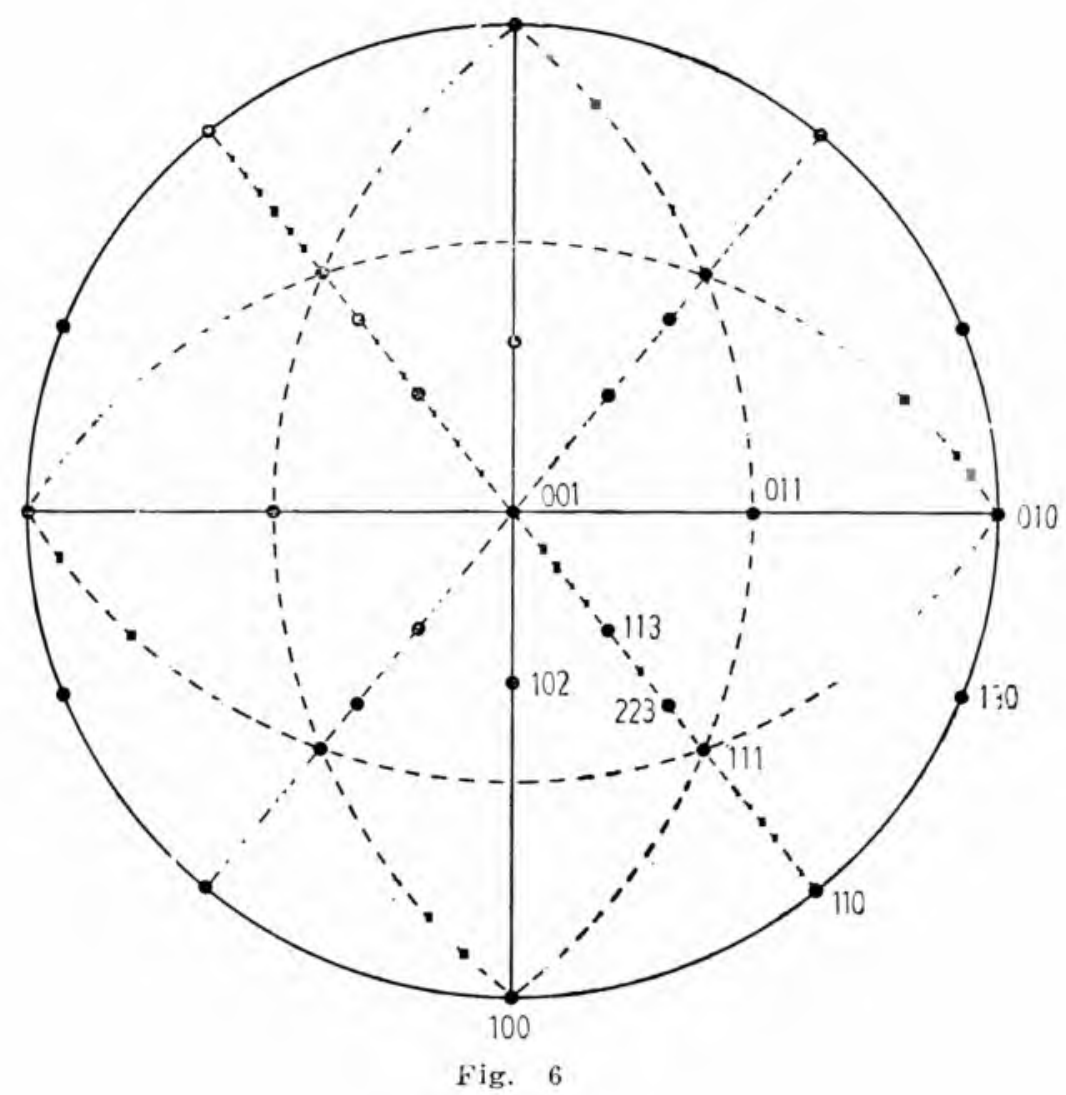

Com relação paramétrica obtida, calculamos os valores da penultima coluna da tabela, que se segue, os quaes são muito próximos dos resultados experimentaes encontrados por nós.

(7) Artini - (B. de Aquadurotal - Italia) - Atti. soc. ital. Sc. Nat. Milan. 42, $101-117$, (1903) Ref. Z. Kryst. 41, 226 (1905). 


\section{TABELA}

\begin{tabular}{|c|c|c|c|c|c|}
\hline \multirow{2}{*}{$\begin{array}{l}\text { Angulos } \\
\text { medidos }\end{array}$} & \multirow{2}{*}{$\begin{array}{l}\text { N.o de } \\
\text { medi- } \\
\text { das }\end{array}$} & \multicolumn{2}{|c|}{ Valores experimentaes } & \multirow{2}{*}{$\begin{array}{l}\text { Valores } \\
\text { calculados }\end{array}$} & \multirow[b]{2}{*}{ Diferença } \\
\hline & & $\begin{array}{l}\text { Limite das me- } \\
\text { didas }\end{array}$ & Medias & & \\
\hline$(100):(110)$ & 5 & $39^{\circ} 22^{\prime} \quad 38^{\circ} 58^{\prime}$ & $39^{\circ} 12^{\prime}$ & $39^{\circ} 11^{\prime} 39^{\prime \prime}$ & $21^{\prime \prime}$ \\
\hline$(100):(130)$ & 7 & $67^{\circ} 46^{\prime} \quad 67^{\circ} 39^{\prime}$ & $67^{\circ} 46^{\prime} 22^{\prime \prime}$ & $67^{\circ} 47^{\prime}$ & $3 \rho^{\prime \prime}$ \\
\hline$(110):(130)$ & 8 & $28^{\circ} 34^{\prime} \quad 28^{\circ} 32^{\prime}$ & $28^{\circ} 33^{\prime}$ & $28^{\circ} 34^{\prime}$ & $1^{\prime}$ \\
\hline$(110):(010)$ & 7 & $50^{\circ} 52^{\prime} \quad 50^{\circ} 46^{\prime}$ & $50^{\circ} 46^{\prime} 28^{\prime \prime}$ & $50^{\circ} 48^{\prime} 15^{\prime \prime}$ & $1^{\prime} 47^{\prime \prime}$ \\
\hline$(110):(111)$ & 12 & $25^{\circ} 43^{\prime} \quad 25^{\circ} 41^{\prime}$ & $25^{\circ} 41^{\prime} 8^{\prime \prime}$ & $25^{\circ} 42^{\prime}$ & $52^{\prime \prime}$ \\
\hline$(110):(113)$ & 4 & $55^{\circ} 17^{\prime} \quad 55^{\circ} 12^{\prime}$ & $55^{\circ} 17^{\prime} 32^{\prime \prime}$ & $55^{\circ} 17^{\prime} 52^{\prime \prime}$ & $20^{\prime \prime}$ \\
\hline$(113):(223)$ & 5 & $19^{\circ} 34^{\prime} \quad 19^{\circ} 28^{\prime}$ & $19^{\circ} 29^{\prime} 45^{\prime \prime}$ & $19^{\circ} 28^{\prime}$ & $i^{\prime} 45^{\prime \prime}$ \\
\hline$(111):(223)$ & 5 & $10^{\circ} 12^{\prime} \quad 09^{\circ} 58^{\prime}$ & $10^{\circ} 8^{\prime} 21^{\prime \prime}$ & $10^{\circ} 8^{\prime} 20^{\prime \prime}$ & $1^{\prime \prime}$ \\
\hline$(001):(113)$ & 8 & $34^{\circ} 42^{\prime} \quad 34^{\circ} 39^{\prime}$ & $34^{\circ} 41^{\prime} 30^{\prime \prime}$ & $34^{\circ} 41^{\prime}$ & $30^{\prime \prime}$ \\
\hline$(001):(011)$ & 21 & $52^{\circ} 44^{\prime} \quad 52^{\circ} 39^{\prime}$ & $52^{\circ} 41^{\prime} 30^{\prime \prime}$ & $5 \Sigma^{\circ} 41^{\prime} 30^{\prime \prime}$ & 0 \\
\hline$(1001):(102)$ & 12 & $38^{\circ} 53^{\prime} \quad 38^{\circ} 50^{\prime}$ & $38^{\circ} 50^{\prime} 50^{\prime \prime}$ & $38^{\circ} 49^{\prime} 25^{\prime \prime}$ & $1^{\prime} 25^{\prime \prime}$ \\
\hline$(10 \bar{z}):(102)$ & 1 & - & $77^{\circ} 35^{\prime}$ & $77^{\circ} 37^{\prime}$ & $2^{\prime}$ \\
\hline$(110):(011)$ & 1 & - & $62^{\circ} 19^{\prime}$ & $62^{\circ} 20^{\prime} 40^{\prime \prime}$ & $1^{\prime} 40^{\prime \prime}$ \\
\hline$(100):(102)$ & 3 & $51^{\mathrm{c}} 13^{\prime}$ & $51^{\circ} 11^{\prime} 30^{\prime \prime}$ & $51^{\circ} 11^{\prime}$ & $30^{\prime \prime}$ \\
\hline
\end{tabular}

Aumentando os dados cristalograficos sobre a baritina do Araxá, damos a seguir os indices de refração calculados com a luz amarela do sodio. O processo adotado foi o do "angulo de desvio mi-

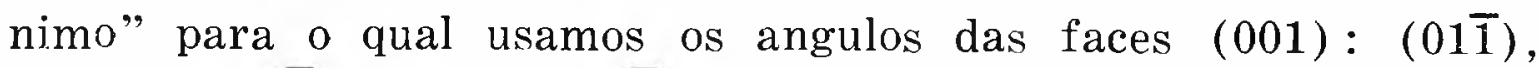
$(001):(10 \overline{2})$ e $(100):(\overline{1} 10)$. Com o primeiro angulo $\left(52^{\circ} 43^{\prime}\right)$

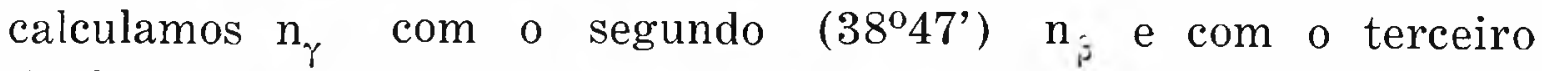
$\left(39^{\circ} 12^{\prime}\right) \mathrm{n}_{\varkappa \text {. }}$

$$
\begin{aligned}
& n_{\gamma}=1,6495 \\
& n_{\gamma}=1,6433 \\
& n_{\alpha}=1,6333
\end{aligned}
$$

Finalmente medimos a angulo dos eixos óticos $2 \mathrm{E}_{\mathrm{a}}=63^{\circ} 9^{\prime} 48^{\prime \prime}$ e utilisando-nos do indice de refração médio calculado achamos o verdadeiro angulo agudo dos eixos óticos $2 \mathrm{~V}_{\mathrm{a}}=37^{\circ} 6^{\prime} 55^{\prime \prime}$ 


\section{SUMMARIUM}

\section{BARITINA}

Baritina ex Araxá crystallis amplitudine variis apparet, quorurn maxima quinque, sex centimetra attingunt.

Color subviridis, interdum flavus. Habitus tabularis ac prismaticus. Vitri modo translucida.

Quas reperimus formas : a $\{100\}, \mathrm{m}\{110\} \mathrm{i}\{130\} \mathrm{b}\{010\}$. o $\{011\} \mathrm{c}\{001\}, \mathrm{v}\{102\}, \mathrm{r}\{111\}, \mathrm{s}\{223\} \mathrm{n}\{113\}$

Relatio parametrica : $\mathrm{a}: \mathrm{b}: \mathrm{c}=0,8156: 1: 1,3123$

Refractionis index : $n_{\alpha}=1,6333 n_{\xi}=1.6433 n_{\gamma}=1,6495$ Opticorum axium angulus : $2 \mathrm{E}_{\mathrm{a}}=63^{\circ} 9^{\prime} 48^{\prime \prime} \quad 2 \mathrm{~V}_{\mathrm{a}}=37^{\circ} 6^{\prime} 55^{\prime \prime}$ 